\title{
Dynamical symmetry approach to path integrals of quantum spin systems
}

\author{
Matouš Ringel $^{1,2}$ and Vladimir Gritsev ${ }^{1,3}$ \\ ${ }^{1}$ Physics Department, University of Fribourg, Chemin du Musée 3, CH-1700 Fribourg, Switzerland \\ ${ }^{2}$ Basler Kantonalbank, Güterstrasse 127 CH-4053 Basel, Switzerland \\ ${ }^{3}$ Institute for Theoretical Physics, Universiteit van Amsterdam, Science Park 904, Postbus 94485, 1098 XH Amsterdam, The Netherlands
}

(Received 18 July 2013; published 16 December 2013)

\begin{abstract}
We develop a dynamical symmetry approach to path integrals for general interacting quantum spin systems. The time-ordered exponential obtained after the Hubbard-Stratonovich transformation can be disentangled into the product of a finite number of the usual exponentials. This procedure leads to a set of stochastic differential equations on the group manifold, which can be further formulated in terms of the supersymmetric effective action. This action has the form of the Witten topological field theory in the continuum limit. As a consequence, we show how it can be used to obtain the exact results for a specific quantum many-body system which can be otherwise solved only by the Bethe ansatz. This represents an example of a many-body system treated exactly using the path-integral formulation. Moreover, our method can deal with time-dependent parameters, which we demonstrate explicitly.
\end{abstract}

DOI: 10.1103/PhysRevA.88.062105

PACS number(s): 03.65.Ca, 71.10.-w, 42.50.-p

\section{INTRODUCTION}

The path-integral approach to strongly correlated systems is a powerful method from many perspectives. In particular, it easily accounts for the topologically nontrivial terms in the action and it is a convenient starting point for various numerical schemes. Moreover, it allows one to treat the correlated systems by a number of approximate analytical techniques, including the saddle-point method, instanton analysis, and various perturbative expansions [1-3]. On the other hand, only a limited number of path integrals are accessible to an exact evaluation. For spin systems the conventional method consists of inserting the resolution of identity on the space of the spin coherent states at every discretized time slice [4]. Then the overlaps between different coherent states taken at consecutive time slices $\langle\zeta(t) \mid \zeta(t+\epsilon)\rangle$, where $\epsilon$ is the discretization time step, is approximated using the Taylor expansion in $\epsilon$. The important assumption behind this is the differentiability of the path $\zeta(t)$. This approximation eventually prohibits exact evaluation of the path integral.

Here we introduce a representation of lattice spin models, which does not rely on the spin coherent state representation, and which reveals a hidden dynamical (super)symmetry structure. After performing the Hubbard-Stratonovich transform, the partition function or the evolution operator of a spin system quadratic in spin variables can be represented as an average of a time-ordered exponential. Based on the well-known facts of the group theory, we represent the time-ordered exponential as a product of the usual exponentials. However, the arguments of the disentangled exponentials are related to the original fields via a set of nonlinear differential equations. These equations can be interpreted as stochastic differential equations. The Hubbard-Stratonovich fields play the role of a noise, whose correlators are defined by the interaction matrix of the ordinal quantum spin system. Stochastic trajectories are nondifferentiable in general and our approach takes this into account exactly and allows one to derive results that can be obtained otherwise only using the Bethe ansatz. We show this explicitly on a nontrivial example rooted in quantum optics [5]. Moreover, the stochastic interpretation suggests that there is a hidden supersymmetry, which eventually leads us to the formulation of the partition function of the quantum spin system as a correlation function of nontopological operators in the theory whose action is given by the topological field theory of Witten [6,7].

Sections II and III of this paper discuss the general aspects of our approach, while Sec. IV illustrates the method on a nontrivial example of a many-body system. We compare our results to the Bethe ansatz solution within the limit of its applicability, and we also provide some explicit results beyond. This example provides a hint of utility of our approach for a larger class of spin systems. The Appendix contains a number of formulas, useful for analytical and numerical considerations.

\section{DISENTANGLEMENT OF THE TIME-ORDERED EXPONENTIAL}

We consider a generic interacting quantum spin model on a lattice,

$$
H=\sum_{i, j} \Omega_{i j}^{a b} S_{i}^{a} S_{j}^{b}+\sum_{j} h_{j}^{a} S_{j}^{a},
$$

where the lattice spin operators $S_{j}^{a}$ ( $j$ being the lattice index) satisfy the commutation relations of the Lie algebra $g,\left[S_{i}^{a}, S_{j}^{b}\right]=f_{c}^{a b} S_{j}^{c} \delta_{i j}$. The indices $a, b, c$ run from 1 to $N$, where $N$ is the dimension of $g$, and $f_{c}^{a b}$ are the structure constants. Interactions between the spins are determined by the interaction matrix $\Omega_{i j}^{a b}$, which can be in general nondiagonal in $a, b$ indices. We assume no particular restriction on the compactness of the corresponding Lie group $G$, while we assume that a certain particular representation is chosen for the concrete physical problem [such as the spin-1/2 or spin-1 representations for the case of $g$ being $\mathrm{SU}(2)]$. We are interested in correlation functions of the model defined by Eq. (1), which is why we introduce the source terms $\mathcal{J}_{j}^{a}(t)$ and consider the generating functional

$$
W[\mathcal{J}]=\operatorname{Tr} \exp \left(i t H+i \sum_{j} \int \mathcal{J}_{j}^{a}(t) S_{j}^{a} d t\right) .
$$


The approach under discussion may work also in a more general setting, in which the matrix $\Omega^{a b}$ and the fields $h^{a}$ are assumed to be time-dependent functions. Moreover, it is straightforward to replace the trace in Eq. (2) by expectation values in certain $\mid$ in $\rangle$ and $\mid$ out $\rangle$. These generalize the approach to nonequilibrium evolution problems. For the equilibrium considerations one can perform the Wick rotation $i t \rightarrow-\beta$.

Applying the Suzuki-Trotter discretization [3] and introducing the Hubbard-Stratonovich transformation [8], we rewrite Eq. (2) into the following form (in the following repeated indices are assumed to be summed over):

$$
W[\mathcal{J}]=\int D \mu(\phi) \operatorname{Tr} \prod_{j} \mathcal{T}_{c} \exp \left(i \int^{t} d t \Phi_{j}^{a} S_{j}^{a}\right),
$$

where

$$
D \mu(\phi)=\exp \left[-\sum_{j k} \int d t \phi_{k}^{a}(t)\left(\Omega^{-1}\right)_{k j}^{a b} \phi_{j}^{b}(t)\right] \prod_{j} D \phi_{j}^{a}
$$

is the Gaussian integration measure, $\Phi_{j}^{a}(t)=\phi_{j}^{a}(t)+h_{j}^{a}+$ $\mathcal{J}_{j}^{a}(t), \Omega^{-1}$ is a matrix inverse to $\Omega$, and $\mathcal{T}_{c} \exp$ denotes the time-ordered exponential. The index $c$ indicates a possible Keldysh contour ordering. The case of time nonlocal action can easily be included into this framework.

The main conceptual step of our approach is the following: the "effective Hamiltonian" $\int d t \Phi_{j}^{a}(t) S_{j}^{a}$ at a given $j$ is a linear combination of generators of $g$ and therefore its exponential is an element of the group $G$. The $\mathcal{T}$-ordered exponential is a direct product of ordinary exponentials, and hence a composition of elements of the Lie group. Any composition of elements of the Lie group $G$ is a certain element of $G$ itself. As such, this element can be written as a single exponential again. This generic mathematical fact defines the essence of our approach and exhibits a concept of dynamical symmetry: a Hamiltonian of the form of a linear combination of generators of the Lie algebra (with possibly time-dependent coefficients) has this Lie algebra as the spectrum-generating algebra [9]. We call the procedure of going from the time-ordered exponential to the product of the ordinary exponentials a disentanglement transformation.

We note that a similar philosophy has been recently used in [10-20]. However, in these papers only one of the many possible representations [namely the one given by Eq. (8) below] has been used. This transformation is then used to perform a direct change of variables in the path integral. Here we present a covariant formulation of the disentanglement transformation. Thus our approach allows us to find a connection with stochastic formulation which then makes the hidden supersymmetry obvious. As a consequence, we reveal a topological field-theory formulation of the quantum spin systems. We therefore do not consider the disentanglement transformation as a direct change of variables. This is another difference with the previously used approach.

It is clear that the original variables $\Phi^{a}(t)$ and a set of variables, which carries out a disentanglement, are related in a nontrivial way. The easiest way to elucidate this relationship is to consider the definition of the $\mathcal{T}$-ordered exponential, namely $i \dot{U}(t)=\Phi^{a}(t) S^{a} U$. Since $U \in G$ and there is an inverse, $U^{-1}$, we write it as $i \dot{U} U^{-1}=\Phi^{a}(t) S^{a}$. The left-hand side of this equation is a current $J_{0}$ on the group, used, e.g., for formulating the nonlinear $\sigma$ models. Group manifold can be parametrized in a number of ways by a set of parameters $\left\{n^{a}\right\},(a=1 \ldots \operatorname{dim} G)$. However, there is an important object-the Maurer-Cartan (MC) one-form on $G$ - that allows us to write the underlying equations in a covariant way. Defining a right one-form $R_{\alpha}^{a}$ via $i \dot{U} U^{-1}=$ $i \partial_{\alpha} U U^{-1} \dot{n}^{\alpha}=i R_{\alpha}^{a}(\{n\}) S_{a} \dot{n}^{\alpha}$, where $n^{\alpha}$ is a parametrization of a group, we arrive at a set of equations defining a relation between $\Phi^{a}(t)$ and a disentangling variables $n^{\alpha}$,

$$
i R_{\alpha}^{a}(\{n\}) \dot{n}^{\alpha}(t)=\Phi^{a}(t)
$$

For a given parametrization $\{n\}$, the MC forms satisfy the MC equations, $\partial_{\alpha} R_{\beta}^{a}-\partial_{\beta} R_{\alpha}^{a}+f_{b c}^{a} R_{\alpha}^{b} R_{\beta}^{c}=0$, which are the defining equations for $R_{\alpha}^{b}$. Here and in Eq. (4) we for generality distinguish the upper and lower indexes, which are manipulated with the Killing-Cartan metrics $g_{\alpha \beta}=R_{\alpha}^{a} R_{\beta}^{b} \operatorname{Tr}\left(S^{a} S^{b}\right)$. Equation (4) can in principle be recast in terms of $\left\{\dot{n}^{a}\right\}$; however, this cannot be done globally if the topology of $G$ is nontrivial [e.g., for the $\mathrm{SU}(2)$ case the sphere cannot be covered by a single map). Also note that for $G$ interpreted as the Riemann manifold, MC forms are proportional to vielbeins (tetrads) $e_{\beta}^{a}$.

We envision at least two general ways to proceed. First, thanks to the Gaussian measure $D \mu(\phi)$ of the HubbardStratanovich fields we can interpret Eq. (4) as a set of stochastic differential equations on the group manifold. Second, one can use Eq. (4) as defining rules for the change of variables in the path integral (3), to go from variables $\Phi^{a}$ to the new variables $n^{a}$ that define a disentanglement transform. Here we elaborate more on the first approach.

For a concrete implementation of this approach we consider first the case of $g=\operatorname{su}(2)$ and come back to a generic discussion later on. As we have said, many different parametrizations for a group element $U$ are possible. These include the Euler angle parametrization, $U_{E}(\alpha, \beta, \gamma)=$ $\exp \left(i \alpha S^{3}\right) \exp \left(i \beta S^{2}\right) \exp \left(i \gamma S^{3}\right)$, the covariant parametrization, $U_{C}(\mathbf{A})=\exp (i \mathbf{A} \cdot \mathbf{S})$, and the Gauss parametrization which can be globally defined on the group manifold [21],

$$
U_{G}\left(\xi_{ \pm}, \xi_{z}\right)=\exp \left(\xi_{+} S^{+}\right) \exp \left(\xi_{z} S^{z}\right) \exp \left(\xi_{-} S^{-}\right)
$$

on which we mostly focus. For the latter parametrization we can derive the following set of equations (in imaginary time):

$$
\begin{aligned}
& \Phi_{-}=\dot{\xi_{+}}-\xi_{+} \dot{\xi}_{z}-\xi_{+}^{2} e^{-\xi_{z}} \dot{\xi}_{-}, \\
& \Phi_{z}=\dot{\xi}_{z}+2 \xi_{+} e^{-\xi_{z}} \dot{\xi}_{-}, \quad \Phi_{+}=e^{-\xi_{z} \dot{\xi}_{-}}
\end{aligned}
$$

and, inversely,

$$
\begin{aligned}
& \dot{\xi}_{+}=\Phi_{-}+\Phi_{z} \xi_{+}-\Phi_{+} \xi_{+}^{2}, \\
& \dot{\xi}_{z}=\Phi_{z}-2 \Phi_{+} \xi_{+}, \quad \dot{\xi}_{-}=\Phi_{+} \exp \left(\xi_{z}\right) .
\end{aligned}
$$

The initial conditions $\xi_{ \pm, z}(0)=0$ follow from the relation $U(0)=1$. For the real time evolution the factor of $i$ should be present in front of $\dot{\xi}_{ \pm, 0}$.

The trace of the evolution operator can be easily computed for the spin-1/2 representation of the evolution operator, $\operatorname{Tr} U_{G}=2 \cosh \left(\xi_{z} / 2\right)+\xi_{+} \xi_{-} \exp \left(-\xi_{z} / 2\right)$. For a generic representation of spin $s$ the covariant form $U_{C}$ yields a compact 
formula for the characters, $\chi^{s}(|\mathbf{A}|)=\sum_{m=-s}^{m=s} \exp (-i m|\mathbf{A}|)=$ $\sin \left[\left(s+\frac{1}{2}\right)|\mathbf{A}|\right] / \sin \left[\frac{1}{2}|\mathbf{A}|\right]$, while for the $\operatorname{su}(N)$ case one can use the celebrated Weyl determinant formula for the characters [22,23].

Next, by looking at Eqs. (7) we realize that only the equation for $\xi_{+}$is independent, while the solutions for $\xi_{z,-}$ can be obtained from the one for $\xi_{+}$. This motivates us to look for a further change of variables. This can be done in different ways. In particular, by introducing new fields $\psi^{ \pm, z}$ via the following correspondence,

$$
\begin{aligned}
& \xi_{+}=\psi^{-}, \quad \xi_{z}=\int_{0}^{t} \psi^{z}\left(t^{\prime}\right) d t^{\prime} \\
& \xi_{-}=\int_{0}^{t} \psi^{+}\left(t^{\prime}\right) \exp \left(\int_{0}^{t^{\prime}} \psi^{z}\left(t^{\prime \prime}\right) d t^{\prime \prime}\right) d t^{\prime}
\end{aligned}
$$

we obtain

$$
\begin{gathered}
\Phi^{z}=\psi^{z}+2 \psi^{+} \psi^{-}, \quad \Phi^{+}=\psi^{+}, \\
\Phi^{-}=\dot{\psi}^{-}-\psi^{z} \psi^{-}-\psi^{+}\left(\psi^{-}\right)^{2} .
\end{gathered}
$$

This representation was found in [12-17] and more recently in $[10,11]$. The measure of integration changes correspondingly, $D \phi^{z} D \phi^{+} D \phi^{-}=\mathcal{M} D \psi^{z} D \psi^{+} D \psi^{-}$, where $\mathcal{M}_{\psi}=$ $c_{\psi} \exp \left[-\frac{1}{2} \int_{0}^{t} \psi^{z}(t) d t\right]$ is the Jacobian of the transformation. The constant $c_{\psi}$ fixes the normalization, and can be adjusted, e.g., comparing to a noninteracting system.

The following change of variables [24],

$$
\begin{aligned}
& \xi_{+}(t)=\rho^{-}(t), \quad \xi_{z}(t)=\int_{0}^{t}\left(\rho^{z}-\rho^{+} \rho^{-}\right) d t^{\prime}, \\
& \xi_{-}(t)=\int_{0}^{t} \rho^{+}\left(t^{\prime}\right) \exp \left[\int_{0}^{t^{\prime}}\left(\rho^{z}-\rho^{+} \rho^{-}\right) d t^{\prime \prime}\right] d t^{\prime},
\end{aligned}
$$

leads to the following equations:

$$
\Phi^{+}=\rho^{+}, \quad \Phi^{-}=\dot{\rho}^{-}-\rho^{-} \rho^{z}, \quad \Phi^{z}=\rho^{z}+\rho^{-} \rho^{+} .
$$

The differential equation for $\dot{\rho}$ can be formally solved,

$$
\begin{aligned}
\rho^{-}(t)= & \exp \left(\int_{0}^{t} \rho^{z}\left(t^{\prime}\right) d t^{\prime}\right) \\
& \times \int_{0}^{t} \exp \left(-\int_{0}^{t^{\prime}} \rho^{z}\left(t^{\prime \prime}\right) d t^{\prime \prime}\right) \Phi^{-}\left(t^{\prime}\right) d t^{\prime},
\end{aligned}
$$

where we assumed the initial condition $\rho^{-}(0)=0$. Therefore, in this representation we implicitly resolved the nonlinearity of the equations in exchange for the nonlocality in time. Variables $\xi_{ \pm, z}$ can be now expressed exactly via $\rho^{z}, \phi^{+}, \phi^{-}$. Therefore, we can use it to construct some mixture representation which involves $\phi$ variables and $\rho^{z}$ simultaneously. This representation suggests that the variable $R(t)=\int_{0}^{t} \rho^{z}(x) d x$ [such that $\dot{R}=\rho^{z}(t)$ ] considered as an independent variable might be useful. The Jacobian in this case reads $\mathcal{M}_{\rho}=$ $c_{\rho} \exp \left[\frac{1}{2} \int_{0}^{t}\left(-\rho^{z}+\rho^{-} \rho^{+}\right)\right]$.

One more example is given by the change of variables

$$
\begin{aligned}
& \xi_{+}(t)=\lambda^{-}(t), \quad \xi_{z}(t)=\int_{0}^{t}\left(\lambda^{z}-2 \lambda^{+} \lambda^{-}\right) d t^{\prime}, \\
& \xi_{-}(t)=\int_{0}^{t} \lambda^{+}\left(t^{\prime}\right) \exp \left[\int_{0}^{t^{\prime}}\left(\lambda^{z}-2 \lambda^{+} \lambda^{-}\right)\right] d t^{\prime \prime},
\end{aligned}
$$

which leads to the following equations:

$$
\begin{aligned}
& \Phi^{+}=\lambda^{+}, \quad \Phi^{-}=\dot{\lambda}^{-}-\lambda^{-} \lambda^{z}+\left(\lambda^{-}\right)^{2} \lambda^{+}, \\
& \Phi^{z}=\lambda^{z} .
\end{aligned}
$$

The advantage of this representation lies in the simplicity of the relationship between the + and $z$ components of the fields. This can be useful for some particular physical situations. The Jacobian of the change of variables from $\phi^{ \pm, z}$ to $\lambda^{ \pm, z}$ is $\mathcal{M}_{\lambda}=c_{\lambda} \exp \left[\frac{1}{2} \int_{0}^{t}\left(-\lambda^{z}+2 \lambda^{-} \lambda^{+}\right)\right]$.

We note that there is an infinite number of other parametrizations, which could be constructed in a similar fashion. Based on the above parametrizations, one could conjecture that the determinant of the transformation in the Gauss decomposition can be expressed in the form of a group element corresponding to the time-averaged Cartan subalgebra.

While in general the Riccati equations encountered above cannot be solved analytically, we note that one can establish an interesting connection to the theory of the KdV (Korteweg-de Vries) equation [25]. Namely, using the projective linear (Möbius) transformation, the Riccati equation can always be put into the form $i y^{\prime}(x)+y^{2}(x)=k^{2}-u(x)$, where $u(x) \equiv$ $u(\Phi(x))$ is a known function of the HS variables $\Phi(x)$, with $x \equiv x(t)$ being a reparametrized time variable and $k$ a real parameter (which could be related to real physical parameters, e.g., magnetic field or $\hbar$ in the WKB series). The function $y(x)$ then admits a series expansion $y(x, k)=$ $k+\sum_{n \geqslant 1} y_{n}(x)(2 k)^{-n}$. Here, all even coefficients $y_{2 n}$ are total derivatives and imaginary, while the first few nontrivial terms are $y_{1}=u(x), y_{3}(x)=u^{2}, y_{5}=u^{3}+\left(u^{\prime}\right)^{2} / 2, \quad y_{7}=$ $5 u^{4} / 2-5 u^{2} u^{\prime \prime} / 2+\left(u^{\prime \prime}\right)^{2} / 2$, etc. The integrals $\int d x y_{2 n+1}$ form the (Poisson) commuting integrals of the KdV equation. It would be interesting to understand the implications of this observation on our path-integral formulation below.

It can be shown that a generalization of the disentangling transformation for other groups-such as $\mathrm{SU}(N)$-is straightforward: the resulting set of equations will have the form of the matrix Riccati equation. On the other hand, a generalization for supergroups is more tricky and should be studied case by case, especially for atypical representations.

\section{STOCHASTIC INTERPRETATION, SUPERSYMMETRY, AND TOPOLOGICAL FIELD-THEORY FORMULATION}

The Hubbard-Stratonovich transformation introduces a Gaussian measure for the fluctuating fields $\phi_{j}^{a}$. This suggests that the differential equations for the disentangling variables $\xi_{ \pm}, x_{z}$ can be interpreted as a set of stochastic differential (generalized Langevin) equations. Generically, solutions of these equations are nondifferentiable paths which should be treated carefully using the appropriate discretization prescriptions (see the Appendix). This leads to the effective Lagrangian formulation (path-integral formulation) and, on the other hand, reveals the hidden supersymmetry structure of the lattice spin systems. Here we establish the connection between our disentanglement procedure and the theory of stochastic differential equations. 


\section{A. Stochastic interpretation}

Provided the MC form (vielbein) is invertible, the disentangling equations can be put into the form

$$
i \dot{n}_{j}^{\alpha}=\left(R_{\alpha}^{a}\right)_{j}^{-1} \Phi_{j}^{a}
$$

for every lattice site $j$. The path-integral formulation of the stochastic processes is a delicate issue and has a long history. The difficulty comes from the proper definition of the continuous-in-time effective Lagrangian from the discretized version of the stochastic processes. This issue was carefully elaborated upon in a series of earlier works [26] (and rediscovered recently in [27]) where it was shown that the result for the effective Lagrangian is a two-parametric family labeled by $r, s \in[0,1]$ which stand for discretization of the stochastic variable and the noise, respectively. While the midpoint discretization $r=s=1 / 2$ corresponds to the Stratonovich convention, the $r=s=0$ is the Ito convention. It is convenient to introduce a multiindex $\mu=(a=1,2,3 ; j=1, \ldots N)$ and rewrite Eq. (15) in the following form,

$$
i \dot{q}^{\mu}=-F^{\mu}(\mathbf{q})+\sigma_{\mu}^{\nu}(\mathbf{q}) \tilde{\phi}^{\mu},
$$

where $q^{\mu}=\left(n^{a}\right)_{j}, F^{\mu}(\mathbf{q})$ is a noise-free (deterministic) part of the equations [which collects the terms containing the magnetic field $h^{a}$ and the source term $\left.\mathcal{J}(t)\right]$, and $\sigma_{v}^{\mu}(\mathbf{q})=$ $\left(R_{\alpha}^{a}\right)_{k}^{-1} O_{k j}$ is a matrix composed of MC forms $\left(R_{\alpha}^{a}\right)_{j}$, and the transformation matrix $O_{k j}$, which puts the noise $\phi_{j}^{a}$ into the form of the Gaussian normalized white noise, $\left\langle\tilde{\phi}^{\mu}(t) \tilde{\phi}^{v}\left(t^{\prime}\right)\right\rangle=\delta^{\mu v} \delta\left(t-t^{\prime}\right)$. This can be always done as soon as the matrix $\Omega_{i, j}^{a, b}$ is nondegenerate. For our lattice spin system the components of the matrix $O_{j k}$ are therefore made of the Fourier coefficients normalized by the Fourier frequencies. The generalized vielbeins $\sigma_{v}^{\mu}$ normalized as $\sigma_{\rho}^{\mu} \sigma_{\mu \nu}=\delta_{\rho \nu}$ define the metric tensor $g_{\mu \nu}=\sigma_{\mu \rho} \sigma_{\nu \rho}$, so that $g^{\mu \nu} g_{v \rho}=\delta_{\rho}^{\mu}$. The effective continuous-in-time Lagrangian then takes the following form:

$$
\begin{aligned}
\mathcal{L}_{r, s}[q, \dot{q}]= & \frac{1}{2} g_{\mu \nu}\left[\dot{q}^{\mu}+F^{\mu}(q)\right]\left[\dot{q}^{\nu}+F^{\nu}(q)\right] \\
& +s \sigma_{\gamma \rho} \partial_{\nu} \sigma_{\rho}^{\nu}\left[\dot{q}^{\gamma}+F^{\gamma}(q)\right]-r \partial_{\mu} F^{\mu}(q) \\
& +\frac{1}{2} s^{2} \partial_{\mu} \sigma_{\rho}^{\nu} \partial_{\nu} \sigma_{\rho}^{\mu} .
\end{aligned}
$$

This general form of the Lagrangian can be simplified for particular physical systems. The integration measure in the path integral reads $D\left(q / \sqrt{\operatorname{det} g_{\mu \nu}}\right)$. This representation is invariant with respect to the change of the particular representation and/or parametrization. The partition function or the time-evolution operator for the lattice spin system is then the expectation value of the operator $O_{N}=\prod_{j} U\left(q_{j}\right)$, $W[\mathcal{J}]=\int D\left(q / \sqrt{\operatorname{det} g_{\mu \nu}}\right) O_{N} \exp \left(-\int d t \mathcal{L}_{r, s}[q, \dot{q}]\right)$.

\section{B. Supersymmetry and connection to topological field theory}

We come back to the general discussion of certain global properties of our approach. We would like to keep all stochastic trajectories without making any approximations. To do this we introduce an identity for every lattice point (a customary trick in field theory [2]),

$$
1=\int D[U] \operatorname{det}(M) \delta\left(J_{0}-\Phi\right),
$$

where we introduced the matrix notations for $\Phi \equiv \Phi^{a} S_{a}$, the current $J_{0}=i \dot{U} U^{-1}$, and the Jacobian $M_{a b}=\delta J_{0} / \delta U_{a b}$ of the transformation. Here $d[U]$ is the Haar invariant integration measure on $G$, satisfying $\int d[U] f(U)=$ $\int d[U] f(U V)$ for any tracelike function $f(U)$. Then $\delta\left(J_{0}-\right.$ $\Phi)=\int d \Lambda /(2 \pi i) \exp \left\{-\operatorname{Tr}\left[\Lambda\left(J_{0}-\Phi\right)\right]\right\}$ (where the integration is performed along the imaginary axis) defines dual fields $\Lambda_{j}^{a}, \Lambda=\Lambda^{a} S_{a}$. Introducing the anticommuting FaddeevPopov ghosts $C^{a}, \bar{C}^{a}$ and using the Grassmann integration, one can write $\operatorname{det} M=\int d \bar{C} d C \exp \left(\bar{C}^{a} M_{a b} C^{b}\right)$ to obtain an effective generating functional after integrating out the stochastic fields,

$$
\begin{aligned}
W[h] & =\int d[U] d \Lambda d \bar{C} d C \exp \left(-\sum_{j} \mathcal{S}_{j}\right) \prod_{j} \operatorname{Tr} U_{j}, \\
\mathcal{S}_{j} & =\operatorname{Tr}\left[\Lambda\left(J_{0}-h\right)\right]_{j}-\bar{C}_{j} D_{0} C_{j}+\operatorname{Tr}\left(\Lambda_{j} \Omega_{j k} \Lambda_{k}\right) .
\end{aligned}
$$

Here $D_{0}=\partial_{t}-J_{0}$ is the covariant derivative in time direction, $j$ is the lattice site index, and $C \equiv\left\{c^{a}\right\}$ represents a vector of Grassman variables. In this representation a generating functional looks like an expectation value of the Polyakov-like string $\prod_{j} \operatorname{Tr} U_{j}$ for a theory described by the action $\sum_{j} S_{j}$. In principle, some of the variables in the effective action can be traced out explicitly. For instance, the variables $\Lambda$ enter quadratically and can be integrated out; the corresponding effective theory would look like the current-current interacting $\sigma$ model coupled to fermions. The Grassmann part can be shown to lead to a finite contribution, which can be computed explicitly. This eventually produces the form (17) of the effective action since the Grassmann variables propagator $\langle\bar{C}(t) C(t)\rangle=s$, where the parameter $0 \leqslant s \leqslant 1$ is the discretization introduced above. The group variables enter the action quadratically into and can be partially [because the expression for $U\left(n^{a}\right)$ is not quadratic] integrated out. However, at this point we would like to keep all the variables as well as their duals to demonstrate an underlying supersymmetry, which is difficult to see otherwise.

The hidden BRST supersymmetry [28] can be traced back to the stochastic nature of differential equations (4) or (7) on the Lie group manifold. In this sense it is a general feature of stochastic equations first noticed in [29,30] (see also [2]). The supersymmetry transformation $\delta$ on variables in (19) is induced by

$$
\begin{gathered}
\delta \Lambda=0, \quad \delta \bar{C}=\epsilon \Lambda, \\
\delta U=\epsilon U C, \quad \delta C=-\epsilon C^{2},
\end{gathered}
$$

and, as a consequence, $\delta J_{0}=\epsilon D_{0} C$ and $\delta\left(D_{0} C\right)=0$. In components it reads $\delta c^{a}=-\frac{1}{2} f_{b c}^{a} \epsilon c^{b} c^{c}$. The action $\sum_{j} \mathcal{S}_{j}$ is therefore BRST exact, $\delta \mathcal{S}=0$. In terms of the group parameters $n^{a}$ the BRST charge can be represented by the action of the operator

$$
\mathcal{Q}=\sum_{j}\left(c^{a} \delta_{n^{a}}+\Lambda_{a} \delta_{\bar{c}^{a}}\right)_{j},
$$


so that $\mathcal{Q}^{2}=0$. Action on any functional is defined by $\left\{\mathcal{Q}, X_{j}\right\}=\mathcal{Q} X_{j}$. One can further show that

$$
\begin{gathered}
\mathcal{S}=\{\mathcal{Q}, V\}, \\
V=\sum_{j} \bar{c}_{j}^{a} J_{0, j}^{a}+\sum_{i, j} \bar{c}_{i}^{a} \Omega_{i j}^{a b} \Lambda_{j}^{b} \\
=\sum_{j} \bar{c}_{j}^{a} J_{0, j}^{a}+\sum_{i j} \Omega_{i j}^{a b}\left\{Q, \bar{c}_{i}^{a}\left\{Q, \bar{c}_{j}^{b}\right\}\right\} .
\end{gathered}
$$

In this form the action is in the form of the topological field theory of Witten [6]. It is easy to see that it is $\mathcal{Q}$ exact. The action of the $\mathcal{Q}$ operator on $U$ is nonzero, and therefore the lattice spin-system partition function is equivalent to computing an expectation value of a product of nonexact operators in this theory. Recently, progress in computing "nontopological"(non-BPS) observables in topological field theories has been made in the framework of instantonic field theory [7]. Concrete applications of these developments to the spin systems shall be discussed elsewhere. We emphasize that this representation of the interacting spin system is exact, since we kept all the nondifferentiable path-integral trajectories exactly.

There are several possible implications of the SUSYtopological field-theory structure. First, there are WardTakahashi identities associated with this supersymmetry. They lead to the fluctuation-dissipation theorem and work relations [31]. Second, these identities allow one to establish renormalization-group prescriptions for the action. In particular, we believe that one can establish the RG flow equations using the generalized tetrads $\sigma_{v}^{\mu}$ in the form of the geometric flow developed in [32]. Third, there is a phenomenon of dimensional reduction [33], coming from two additional Grassmann coordinates in the effective action. Application of these phenomena to spin systems could lead to an interesting relationship between spin systems defined in different dimensions. Fourth, a SUSY breaking pattern is rather restrictive. There are in general three scenarios: unbroken SUSY, breaking on the mean-field level, and dynamical breaking. We believe that one may classify spin systems according to these scenarios. Fifth, the formulation in terms of the topological field theory could shed new light on the origin of the topological order in certain spin systems. Moreover, one of the central concepts of the topological theory, the Witten index, seems to have direct relevance to the sign problem [34].

\section{EXPLICIT DEMONSTRATION OF THE METHOD. COMPARISON WITH THE BETHE ANSATZ AND BEYOND}

We shall illustrate the technique discussed in the previous sections by considering a problem of a single atom interacting with a one-dimensional photonic waveguide, treated within the rotating wave approximation (RWA). This problem has been solved by the Bethe ansatz in [5]; see also [35-37].

\section{A. Model and the observables}

We assume that the photons in the waveguide feature a linear dispersion with two (left,right) branches, described by annihilation operators $a_{R, L}$. For one scatterer only, it is convenient to work in the basis of even (odd) photons $a_{e, o}=\left(a_{R} \pm a_{L}\right) / \sqrt{2}$. Odd photons $a_{o}$ do not interact with the atom, which is why they evolve trivially as free photons. The interacting part of the evolution is hidden in the even component $a_{e}$. From now on we focus purely on this nontrivial part and drop the index $e\left(a_{e} \equiv a\right)$. We consider the interaction between a collection of $M$ atoms, located within a region much smaller than the wavelength of light, and the even component of the photonic field. The atoms may be modeled as a single spin $\hat{\mathrm{S}}$ of magnitude $j=M / 2$. The RWA Hamiltonian of the system then reads $[5,35]$

$$
\hat{\mathrm{H}}=\sum_{k} k a_{k}^{\dagger} a_{k}+\Delta(t) \hat{\mathrm{S}}^{z}+g(t) \sum_{k}\left(\hat{\mathrm{S}}^{+} a_{k}+\hat{\mathrm{S}}^{-} a_{k}^{\dagger}\right) .
$$

We have assumed $k$ independent, but potentially timedependent light-matter coupling $g(t)$, as well as timedependent atomic detuning $\Delta(t)$ (to save space, in the following we drop the explicit time dependence from them).

Many interesting physical properties of this mode may be expressed in terms of specific matrix elements of the evolution operator. For the sake of calculations, we add the auxiliary photonic sources $J_{k}^{(*)}(t)$, which serve the purpose of generating photons in the in and out states. They are set to zero at the end of the calculations. A generic matrix element of the evolution operator in the presence of the photonic sources reads

$$
\mathcal{U}_{o i}:=\left\langle\text { out }\left|\mathcal{T} e^{-i \int_{t_{1}}^{t_{2}} d t H+\sum_{k}\left[J_{k}^{*}(t) a_{k}+J_{k}(t) a_{k}^{\dagger}\right]}\right| \text { in }\right\rangle .
$$

We require that both $\mid$ out $\rangle$ and $\mid$ in $\rangle$ are tensor products of vacuum state within the photonic subspace and a certain spin state. For instance, the amplitude of the decay of a fully excited state $\left(s^{z}=M / 2\right)$ into $2 j$ photons can be related to Eq. (26) as

$$
\begin{aligned}
T_{k_{1}, \ldots, k_{2 j}} & :=\left\langle k_{1}, \ldots, k_{2 j} ;-j|\mathcal{U}(\infty, 0)| 0 ; j\right\rangle \\
& =\frac{\partial^{2 j}}{\partial J_{k_{1}}^{*} \cdots \partial J_{k_{2 j}}^{*}}\left\langle-j\left|\mathcal{T} e^{-i \int_{0}^{\infty} d t H}\right| j\right\rangle .
\end{aligned}
$$

Also the problem of finding the scattering matrix of $n$ photons scattering on the atoms can be reduced to Eq. (26) using the LSZ formalism [37,38]. This formalism expresses the $T$ matrices of the scattering

$i T_{k_{i} \rightarrow p_{i}}=\left.(2 \pi)^{n} G^{(2 n)}\left(\left\{k_{i}\right\},\left\{p_{i}\right\}\right) \prod_{s=1}^{n}\left[\mathcal{G}_{0}^{-1}\left(k_{s}\right) \mathcal{G}_{0}^{-1}\left(p_{s}\right)\right]\right|_{\text {onshell }}$

via the $n$-particle Green function, calculated as

$$
G^{(2 n)}=\frac{(-1)^{n} \delta^{2 n} \ln Z\left[J, J^{*}\right]}{\delta J_{1} \cdots \delta J_{n} \delta J_{1}^{*} \cdots J_{n}^{*}},
$$

where $Z\left[J, J^{*}\right]$ is the generating functional of the Green functions [3] defined by the equation

$$
Z\left[J, J^{*}\right]=\left\langle-j\left|\mathcal{T} e^{-i \int_{-\infty}^{\infty} d t H+\sum_{k}\left[J_{k}^{*}(t) a_{k}+J_{k}(t) a_{k}^{\dagger}\right]}\right|-j\right\rangle .
$$

This obviously forms a special case of Eq. (26).

\section{B. Implementing disentangling transform and the stochastic formulation}

We may treat the amplitude (26) within the path-integral approach. We rewrite the amplitude as a coherent-state path 
integral over the photonic variables, while we retain the time ordering for the spin variables [3],

$$
\begin{aligned}
\mathcal{U}_{o i}= & \int \mathcal{D}\left[a, a^{*}\right]\langle\text { out }| \mathcal{T} e^{i \int d t \sum_{k} a_{k}^{*}\left(i \partial_{t}-k\right) a_{k}} \\
& \times e^{i \int d t\left\{\sum_{k}\left[-g\left(a_{k}^{*} \hat{\mathrm{S}}^{-}+a_{k} \hat{\mathrm{S}}^{+}\right)+J_{k}^{*} a_{k}+J_{k} a_{k}^{*}\right]-\Delta \hat{\mathrm{S}}^{z}\right\}}|\mathrm{in}\rangle .
\end{aligned}
$$

The integral over the bosonic degrees of freedom may be performed explicitly and gives rise to a spin model with an effective spin-spin interaction. Thanks to the linearity of the photonic spectrum, this spin-spin interaction happens to be local in time, which simplifies the calculations enormously. After some algebraic manipulations we arrive at the expression

$$
\begin{aligned}
& \mathcal{U}_{o i}=\mathcal{U}_{o i}^{0}\langle\text { out }| \mathcal{T} e^{\int d t-\frac{|g|^{2}}{2} \hat{S}^{2}} e^{\int d t\left\{\frac{|g|^{2}}{2} S^{2}-\left[i \Delta+\frac{|g|^{2}}{2}\right] S^{z}+\mathcal{J} S^{+}+\mathcal{J}^{*} S^{-}\right\}} \\
& \times \mid \text {in }\rangle \text {, }
\end{aligned}
$$

with

$$
\mathcal{J}^{\mp}(t):=g(t) \sum_{k} \int_{0}^{\infty} d \tau J_{k}^{(*)}(t \mp \tau) e^{-i k \tau} .
$$

The symbol $\mathcal{U}_{o i}^{0}$ denotes the free-photonic-evolution part of the amplitude, which is irrelevant for the problems under consideration and consequently can be ignored. As explained in the first part of this paper, the natural way of decoupling the spin-spin interaction is via the Hubbard-Stratonovich transform (local in time) [8]. The decoupling converts the exponent in Eq. (32) into a linear form, disentangleable using Eq. (5), at the expense of introducing a real-valued white-noise field $\Phi(t)$ :

$$
\begin{aligned}
\mathcal{U}_{o i}= & \mathcal{U}_{o i}^{0}\left\langle\langle\text { out }| \mathcal{T} e^{\int d t-\frac{|g|^{2}}{2} S^{2}}\right. \\
& \left.\left.\times e^{\int d t\left\{\left[g \Phi-i \Delta-\frac{|g|^{2}}{2}\right] S^{z}+\mathcal{J}^{-} S^{+}+\mathcal{J}^{+} S^{-}\right\}} \mid \text {in }\right\rangle\right\rangle_{\Phi},
\end{aligned}
$$

where we define the white-noise averaging $\langle\cdots\rangle_{\Phi}$ to be [39]

$$
\langle\mathcal{A}[\Phi]\rangle_{\Phi}=\frac{\int \mathcal{D}[\Phi] e^{-\frac{1}{2} \int d t \Phi^{2}(t)} \mathcal{A}[\Phi]}{\int \mathcal{D}[\Phi] e^{-\frac{1}{2} \int d t \Phi^{2}(t)}} .
$$

The evolution operator in Eq. (34) can be now readily disentangled using the technology explained in the first part of this paper. The evolution of the new variables $\xi_{ \pm, z}$ is governed by a specific form of Eqs. (7), which we converted into the Ito form [40]

$$
\begin{aligned}
& \dot{\xi}_{+}=\mathcal{J}^{-}-i \Delta \xi_{+}-\mathcal{J}^{+} \xi_{+}^{2}+|g| \xi_{+} \Phi, \\
& \dot{\xi}_{z}=-\left[i \Delta+|g|^{2} / 2\right]-2 \mathcal{J}^{+} \xi_{+}+|g| \Phi, \\
& \dot{\xi}_{-}=\mathcal{J}^{+} e^{\xi_{z}} .
\end{aligned}
$$

These equations form a set of nonlinear stochastic differential equations (SDE) in the Ito form [40,41]. Because of their nonlinearity, it is not possible to write down their explicit solution. We may, however, express the quantities of interest in terms of $\xi_{ \pm, z}$ and write down the underlying SDE for these, utilizing the Ito lemma [40,42]. Averaging these with respect to the noise $\Phi$ we get rid of the noise term and arrive at ordinary differential equations for the averages. Inspecting the matrix elements of Eq. (27) and Eq. (30) and comparing them to the disentangling transformation of Eqs. (7), we conclude that these are straightforwardly related to the $\Phi$ averages of the functions $\xi_{-}^{2 j} e^{-j \xi_{z}}$, or $e^{-j \xi_{z}}$, respectively. The Ito lemma applied to these, however, leads to unclosed equations, because extra powers of $\xi_{ \pm}$pop up. This obstacle may be overcome by considering a more general problem, in particular a hierarchy of equations for the functions $\xi_{+}^{n} e^{-j \xi_{z}}$, or $\xi_{-}^{n} e^{(j-n) \xi_{z}}$, respectively. This includes the desired quantities as a special case. Averaging these equations as indicated above, we obtain a closed set of ordinary differential equations for the following averages,

$$
F_{n}:=\left\langle\xi_{+}^{n} e^{-j \xi_{z}}\right\rangle_{\Phi}, \quad R_{n}:=\left\langle\xi_{-}^{n} e^{(j-n) \xi_{z}}\right\rangle_{\Phi},
$$

with $0 \leqslant n \leqslant 2 j$. The physically interesting quantities stem from $F_{0}$ and $R_{2 j}$, respectively. These auxiliary quantities satisfy by definition the initial conditions $F_{0}\left(t_{1}\right)=1$ and $F_{n>0}\left(t_{1}\right)=0$ (the same for $R_{n}$ ). Based on Eqs. (36) the quantities $F_{n}$ and $R_{n}$ satisfy a simple system of linear ordinary differential equations (the noise has been already averaged out),

$$
\begin{aligned}
\dot{F}_{n}= & {\left[i \Delta(j-n)+\frac{g^{2}}{2}[j(j+1)-2 j n+n(n-1)]\right] F_{n} } \\
& +n \mathcal{J}^{-} F_{n-1}+(2 j-n) \mathcal{J}^{+} F_{n+1}, \\
\dot{R}_{n}= & {\left[-i \Delta(j-n)-\frac{g^{2}}{2}(2 j-n)(n+1)\right] R_{n}+\mathcal{J}^{+} n R_{n-1} . }
\end{aligned}
$$

These equations contain all information necessary to describe the two underlying physical problems.

\section{Scattering of $\boldsymbol{n}$ photons: Results}

According to Eq. (30) and Eq. (37) we can establish that all that is needed to calculate the scattering matrix for any number of incoming photons is hidden in the logarithm of the generating functional $Z$ of Eq. (30), which up to terms that do not contribute to the scattering matrices reads $\ln Z\left[J, J^{*}\right]=$ $\left.\ln F_{0}(\infty)\right|_{t_{1}=-\infty}$. It is now essential to realize that we can treat $\ln Z$ perturbatively in the sources $J^{(*)}$. According to the LSZ reduction formula (28), the scattering of $n$ photons is fully and exactly described by the $2 n$th term of the Taylor expansion of $\ln Z$ with respect to the sources $J^{(*)}$. We introduce a control expansion parameter $\lambda$, such that $J^{(*)}=O(\sqrt{\lambda})$, and calculate the expansion of $Z[J, J *]=1+a_{1}+a_{2}+\cdots$ with the coefficients $a_{n}=O\left(\lambda^{n}\right)$. The power series of $\ln Z$ can be then written as $\ln \left(1+a_{1}+a_{2}+a_{3}+\cdots\right)=a_{1}+\left(a_{2}-\frac{a_{1}^{2}}{2}\right)+\left(a_{3}-\right.$ $\left.a_{1} a_{2}+\frac{a_{1}^{3}}{3}\right)+\cdots$, which we organized order by order.

Solving the hierarchy (38) up to fourth order in $J^{(*)}$, we deduce the $T$ matrices for one- and two-photon scattering $T^{(1,2)}$,

$$
i T_{k \rightarrow p}^{(1)}=\delta(p-k) \frac{-2 i j g^{2}}{k+i j g^{2}},
$$

and

$$
\begin{aligned}
i T_{k_{1,2} \rightarrow p_{1,2}}^{(2)}= & \frac{\delta\left(E-E^{\prime}\right) \times 16 g^{4} i j E}{\left[E+i g^{2}(2 j-1)\right]} \\
& \times \frac{\left(E+2 i j g^{2}\right)}{\left[\left(E+2 i j g^{2}\right)^{2}-\Delta^{2}\right]\left[\left(E+2 i j g^{2}\right)^{2}-\Delta^{\prime 2}\right]} .
\end{aligned}
$$


After transforming the even (odd) basis back to the left (right) basis, one may check that these results completely agree with those calculated using the Bethe ansatz in $[5,35]$.

\section{Decay of an initially excited state}

Let us investigate the problem of the decay of an initial fully excited state. At $t_{1}=0$ all the emitters are supposed to be in the upper state, which corresponds to the highest weight spin state with $s_{z}=j$. The emitters consequently relax into the ground state and radiate outgoing photons. A conserved quantity - the number of excitations-fixes the number of outgoing photons to be $2 j$-the number of atoms in the cluster. Equations (27), (37), and (39) allow us to find an exact solution in quadratures for general sources $J^{(*)}$. For the time-independent coupling $g$ and detuning $\Delta$ the underlying integrals can be performed analytically. For instance, we may derive the spectral distribution of the outgoing photons, namely the single-photon spectrum

$$
\mathcal{P}_{2 j}(q):=\int \prod d k_{m}\left(\sum_{m} \delta\left(q-k_{m}\right)\right)\left|T_{k_{1}, \ldots, k_{2 j}}\right|^{2} .
$$

An explicit calculation gives a normalized result,

$$
\mathcal{P}_{2 j}(q)=\sum_{m=0}^{2 j-1} \frac{1}{2 j \pi} \frac{g^{2}\left[j(j+1)-(j-m)^{2}\right]}{g^{4}\left[j(j+1)-(j-m)^{2}\right]^{2}+(q-\Delta)^{2}},
$$

where each of the terms is a normalized Lorentz curve with the peak at $q=\Delta$ and the width $j(j+1)-(j-m)^{2}$.

\section{E. Time-dependent parameters}

Time-dependent parameters $\Delta(t)$ and $g(t)$ do not pose any fundamental obstacle for our method; it is just not possible to obtain the results analytically. The time-dependent regime is, however, inaccessible to the Bethe ansatz solution, which suggests superiority of the technique proposed here. On the other hand, a periodic driving of the coupling constant is of direct physical interest, for instance within the cavity-QED setups $[43,44]$.

For illustration, we treat the hierarchy (39) describing the decay of an excited state numerically. The solution stabilizes quickly with growing $t_{2}$ and it is thus enough to retain only a finite time $t_{2}$ to extract the limit $t_{2} \rightarrow \infty$. We explicitly demonstrate the effect of time-dependent parameters in the case of a two-level system $(j=1 / 2)$. We let the coupling constant $\Gamma(t)=|g(t)|^{2}$ oscillate as $\Gamma(t)=\Gamma[1+a \cos (\Omega t+$ $\phi)]$ and the atomic level splitting $\Delta(t)$ oscillate as $\Delta(t)=$ $\Delta_{0} \cos (\omega t+\varphi)$. Note that the amplitude depends on the initial phases $\phi, \varphi$. As in experiment they may or may not be fixed; we present also a probability distribution averaged over possible initial phases. The results of our numerical calculation are presented in Figs. 1 and 2. The oscillating coupling constant leads to an emergence of satellite peaks centered at $\pm \Omega$, whose strength decreases with a decreasing modulation depth $a$. When $\Omega \lesssim 1$ these satellite peaks are masked due to averaging over the initial phases, but they survive the averaging when $\Omega$ is larger.
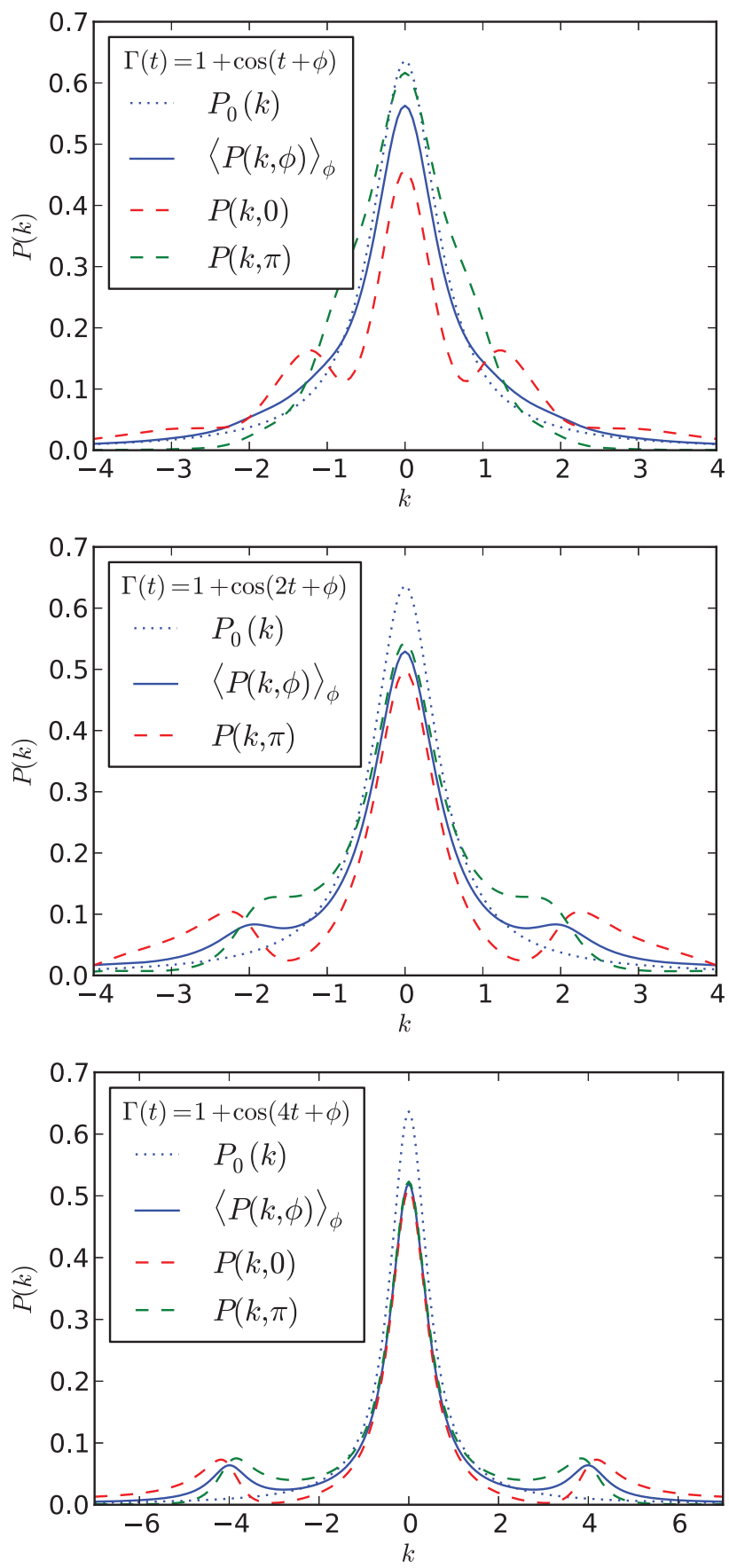

FIG. 1. (Color online) Two-level system decays into one photon. We plot the normalized probability distribution function $P(k)$ of the photon's momentum $k$ (measured in units of $\Gamma=g^{2}$ ), given by Eq. (42) with $j=1 / 2$, i.e. $P(k)=\mathcal{P}_{1}(k)$. The coupling strength $\Gamma$ is assumed to be time dependent, in particular oscillating, while the effective level-splitting $\Delta$ is set to zero. The probability distribution depends on the initial phase $\phi$ of $\Gamma(t)$. We plot $P(k)$ averaged over $\phi$, its value for $\phi=0, \pi$, and for comparison also the $\Gamma(t)=$ const $=1$ probability distribution $P_{0}(k)$. Note that the oscillation $\Gamma(t)=1+\cos (\Omega t+\phi)$ creates an additional peak at $k= \pm \Omega$.

\section{DISCUSSION AND CONCLUSION}

In this paper we discussed a path-integral formulation for the lattice spin systems using the disentanglement procedure of the time-ordered exponential. This approach avoids using 

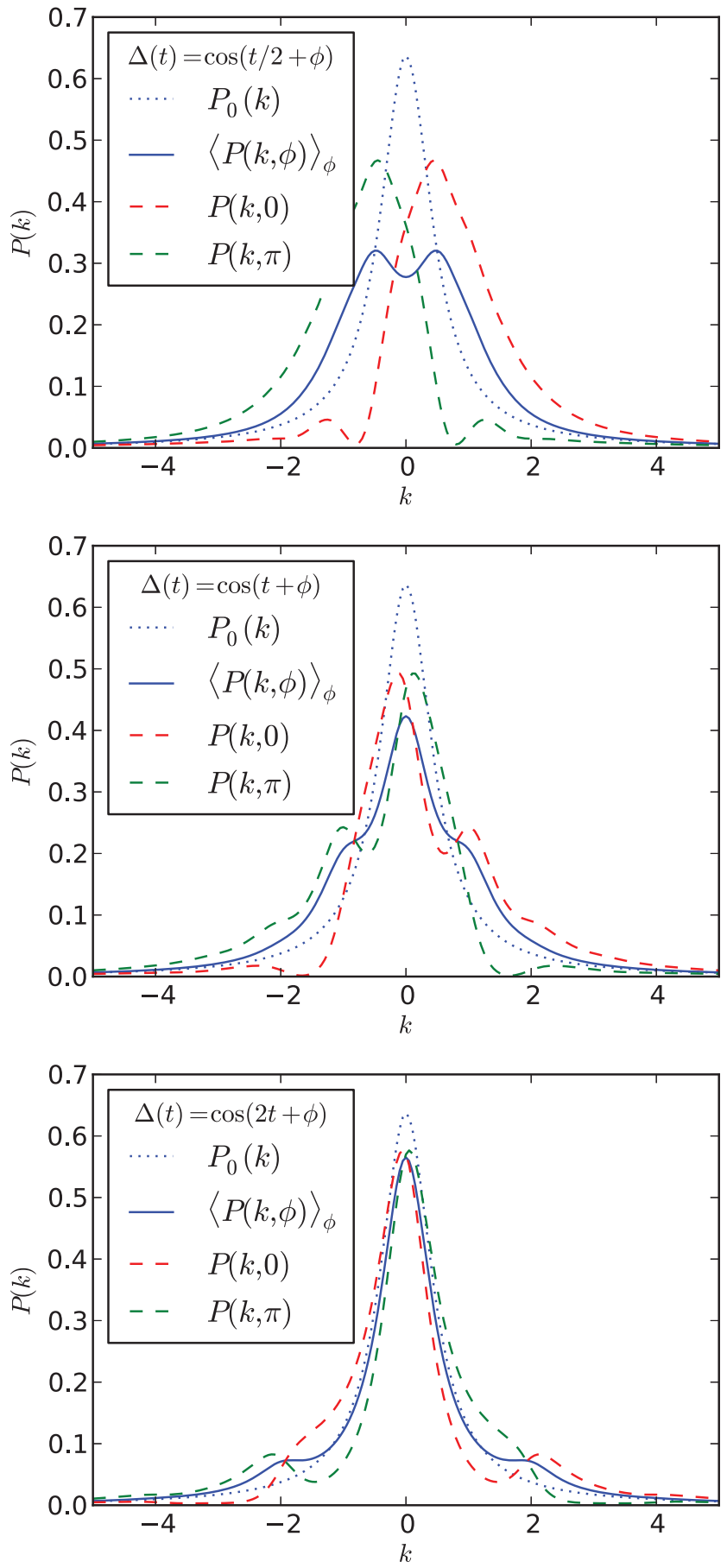

FIG. 2. (Color online) Two-level system decays into one photon. We plot the normalized probability distribution function $P(k)$ of the photon's momentum $k$ (measured in units of $\Gamma=g^{2}$ ), given by Eq. (42) with $j=1 / 2$, i.e., $P(k)=\mathcal{P}_{1}(k)$. The level-splitting $\Delta$ (measured in units of $\Gamma$ ) is assumed to be time dependent, in particular oscillating, while the coupling constant $g$ is assumed to be constant. The probability distribution depends on the initial phase $\phi$ of $\Delta(t)$. We plot $P(k)$ averaged over $\phi$, its value for $\phi=0, \pi$, and for comparison also the $\Delta(t)=$ const $=1$ probability distribution $P_{0}(k)$.

coherent states and is therefore free of their drawbacks. We invoked the stochastic formulation of the disentanglement procedure which effectively keeps all the nondifferentiable paths, and thus is in principle exact. This allowed us to reproduce known results for a Bethe ansatz solvable model. Moreover, we used the method to describe time-dependent parameters where the Bethe ansatz fails to provide a solution. From the fundamental point of view, the stochastic approach to the disentanglement equations reveals a hidden supersymmetric structure of the interacting spin system, and as a consequence leads to the effective action formulation in terms of the topological field theory. We believe that these formulations could shed light on hidden topological and dynamical aspects of interacting lattice spin systems.

We comment here on possible further developments of our representation. The Riccati equation which plays a central role in the procedure has dynamical sl(2) symmetry which could possibly help to select certain orbits in the path integral. For systems defined on higher rank algebras, such as $\operatorname{su}(n)$, this equation becomes a matrix Riccati equation. The effective action in the stochastic formulation can be studied using both nonperturbative and perturbative techniques. Many of these approaches have been developed in the context of OnsagerMachlup theory for nonequilibrium stochastic processes; see, e.g., [2,31]. On the other hand, our very preliminary experience on numerical implementation of the method for a generic spin system shows that large deviations [45] will be an important obstacle to the numerical sampling of stochastic paths.

One more interesting question is how the integrability of certain lattice spin models (like, e.g., $X Y Z$ spin chains) can be uncovered via our path-integral formulation. We envision interesting developments on this route.

\section{ACKNOWLEDGMENTS}

We would like to thank Eugene Demler, Victor Galitski, Mikhail Kiselev, and Mikhail Pletyukhov for many useful discussions and suggestions. This work was supported by the Swiss NSF.

\section{APPENDIX}

In this appendix we collect some relevant information about several parametrizations of the SU(2) group and the related Riemann and differential geometry notions mentioned in the main text.

\section{Different parametrizations of $\mathrm{SU}(2)$}

Here we overview various parametrizations of the group SU(2) and present an explicit form of differential-geometric structures used in the main body of the paper.

The Cayley-Klein parametrization

$$
U(\alpha, \beta)=\left(\begin{array}{cc}
\alpha & \beta \\
-\bar{\beta} & \bar{\alpha}
\end{array}\right)
$$

where $|\alpha|^{2}+|\beta|^{2}=1$. This allows one to introduce new parameters $x_{1, \ldots, 4}, \alpha=x_{1}+i x_{2}$, and $\beta=x_{3}+i x_{4}$, which define an embedding of $S^{3} \simeq \mathrm{SU}(2)$ to $R^{4}$. The underlying equations read

$$
\begin{gathered}
\phi_{3}=i(\dot{\alpha} \alpha+\dot{\beta} \beta), \\
\phi_{+}=i(-\dot{\alpha} \beta+\dot{\beta} \alpha),
\end{gathered}
$$


and the complex conjugates of these two equations. Inverting them (when the determinant of the transformation is nonzero) yields

$$
\begin{aligned}
& i \dot{\alpha}=\alpha \phi_{3}-\phi_{+} \bar{\beta}, \\
& i \dot{\beta}=\phi_{3} \beta+\phi_{+} \bar{\alpha} .
\end{aligned}
$$

The Euler parametrization is defined as

$$
U(\alpha, \beta, \gamma)=e^{i \alpha(t) S^{3}} e^{i \beta(t) S^{2}} e^{i \gamma(t) S^{3}} .
$$

Here $0 \leqslant \alpha<2 \pi, 0 \leqslant \beta \leqslant \pi$, and $0 \leqslant \gamma \leqslant 4 \pi$. The stochastic equations take the following form [18]:

$$
\begin{aligned}
\dot{\alpha} & =-\phi_{1}(t) \cos \alpha \cot \beta-\phi_{2}(t) \sin \alpha \cot \beta+\phi_{3}(t), \\
\dot{\beta} & =-\phi_{1}(t) \sin \alpha+\phi_{2}(t) \cos \alpha, \\
\dot{\gamma} & =\phi_{1}(t) \frac{\cos \alpha}{\sin \beta}+\phi_{2}(t) \frac{\sin \alpha}{\sin \beta} .
\end{aligned}
$$

A rotationally invariant (covariant) parametrization is given by

$$
U(t)=\exp (-i \psi \mathbf{n} \cdot \mathbf{S}),
$$

where $\mathbf{n}(\theta, \phi)$ denotes a unit vector spanning a sphere. Its connection to the Euler parametrization is obtained easily by employing the lowest nontrivial representation-the Pauli matrix parametrization. It may be written as

$$
\begin{gathered}
\phi=\frac{1}{2}(\pi+\alpha-\gamma), \quad \tan \theta=\frac{\tan (\beta / 2)}{\sin \left[\frac{1}{2}(\alpha+\gamma)\right]}, \\
\cos \psi=2 \cos ^{2}\left(\frac{\beta}{2}\right) \cos ^{2}\left(\frac{1}{2}(\alpha+\gamma)\right)-1 .
\end{gathered}
$$

In this representation we define a vector $\mathbf{A}$ with three components $\left(A^{1}, A^{2}, A^{3}\right)$ (don't mix them up with parameters $A, B, C$ in the text for the other representation) by requiring that

$$
U(t)=\exp [i \boldsymbol{\sigma} \cdot \mathbf{A}(t)]
$$

is the disentangled version of the $T$-ordered exponential. Using the standard formulas for the Pauli matrices we obtain

$$
U(t)=\cos |A|+i \frac{\sin |A|}{|A|}(\boldsymbol{\sigma} \cdot \mathbf{A}),
$$

where $|A|$ is the length of the vector $\mathbf{A}$. Considering the time derivative $\partial_{t} U(t)$ and multiplying it by $U^{-1}(t)$, we get

$$
\begin{aligned}
-i U^{-1} \partial_{t} U(t)= & \frac{|A|-\cos |A| \sin |A|}{|A|^{2}} \partial_{t}|A|(\boldsymbol{\sigma} \cdot \mathbf{A}) \\
& +\frac{\cos |A| \sin |A|}{|A|}\left(\boldsymbol{\sigma} \cdot \partial_{t} \mathbf{A}\right) \\
& +\frac{\sin ^{2}|A|}{|A|^{2}}\left[\boldsymbol{\sigma} \cdot\left(\mathbf{A} \times \partial_{t} \mathbf{A}\right)\right],
\end{aligned}
$$

which is to be then identified with the effective Hamiltonian $\boldsymbol{\phi}(t) \cdot \boldsymbol{\sigma}$. We therefore get a vector Langevin equation,

$$
\begin{aligned}
\boldsymbol{\phi}= & \frac{|A|-\cos |A| \sin |A|}{|A|^{2}} \partial_{t}|A| \mathbf{A} \\
& +\frac{\cos |A| \sin |A|}{|A|} \partial_{t} \mathbf{A}+\frac{\sin ^{2}|A|}{|A|^{2}}\left(\mathbf{A} \times \partial_{t} \mathbf{A}\right) .
\end{aligned}
$$

Note also that $(\boldsymbol{\phi} \cdot \mathbf{A})=\frac{1}{2} \partial_{t}|A|^{2}$. From this form it follows that the leading terms of a power expansion in terms of $|A|$ $\operatorname{read} \boldsymbol{\phi}=\partial_{t} \mathbf{A}+\mathbf{A} \times \partial_{t} \mathbf{A}+\cdots$.
The connection between different representations can be obtained by comparing their matrix forms with the CayleyKlein one:

$$
\begin{gathered}
\alpha=\cos \frac{|\mathbf{A}|}{2}+i \frac{A^{z}}{|\mathbf{A}|} \sin |\mathbf{A}|, \\
\beta=i \frac{A_{x}-i A_{y}}{|\mathbf{A}|} \sin |\mathbf{A}|,
\end{gathered}
$$

while, for the Gaussian parametrization, $\exp (-B / 2)=\bar{\alpha}$.

\section{a. Various disentangling formulas}

Let $S_{ \pm, 0}$ be the generators of $\mathrm{su}(2)$ or $\mathrm{su}(1,1)$, so that $\left[S_{-}, S_{+}\right]=2 \sigma S_{0}$ and $\left[S_{0}, S_{ \pm}\right]= \pm S_{ \pm}$, where $\sigma=1$ for the $\operatorname{su}(1,1)$ and $\sigma=-1$ for the $\operatorname{su}(2)$. The Casimir invariant is therefore $C_{2}=S_{0}^{2}-\frac{\sigma}{2}\left(S_{+} S_{-}+S_{-} S_{+}\right)$. The evolution operator can be written in a variety of forms distinguished by a different ordering of terms containing $S_{ \pm, 0}$. These include the symmetric form,

$$
U_{S}=\exp \left(a_{+} S_{+}+a_{0} S_{0}+a_{-} S_{-}\right),
$$

the normal-ordered Cartan form,

$$
U_{N}=\exp \left(A_{+} S_{+}\right) \exp \left(\ln \left[A_{0}\right] S_{0}\right) \exp \left(A_{-} S_{-}\right),
$$

or the anti-normal-ordered Cartan form,

$$
U_{A}=\exp \left(B_{-} S_{-}\right) \exp \left(\ln \left[B_{0}\right] S_{0}\right) \exp \left(B_{+} S_{+}\right) .
$$

They are related as follows [46]:

$$
\begin{aligned}
A_{ \pm} & =\frac{\frac{a_{ \pm}}{D} \sinh D}{\cosh D-\frac{a_{0}}{2 D} \sinh D}, \\
A_{0} & =\left(\cosh D-\frac{a_{0}}{2 D} \sinh D\right)^{2}, \\
B_{ \pm} & =\frac{\frac{a_{ \pm}}{D} \sinh D}{\cosh D+\frac{a_{0}}{2 D} \sinh D}, \\
B_{0} & =\left(\cosh D+\frac{a_{0}}{2 D} \sinh D\right)^{2}
\end{aligned}
$$

and

$$
\begin{array}{ll}
A_{0}=\frac{B_{0}}{\left(1-\sigma B_{+} B_{-} B_{0}\right)^{2}}, & A_{ \pm}=\frac{B_{ \pm} B_{0}}{1-\sigma B_{+} B_{-} B_{0}}, \\
B_{0}=\frac{\left(A_{0}-\sigma A_{+} A_{-}\right)^{2}}{A_{0}}, & B_{ \pm}=\frac{A_{ \pm}}{A_{0}-\sigma A_{+} A_{-}},
\end{array}
$$

where $D=\frac{1}{2}\left(a_{0}^{2}-4 \sigma a_{+} a_{-}\right)^{1 / 2}$.

The ordered product of an arbitrary number of symmetric operators, $U_{S}(n)=\exp \left[a_{+}(n) J_{+}+a_{0}(n) J_{0}+a_{-}(n) J_{-}\right]$ labeled by some discrete index $n=1, \ldots, N$,

$$
U_{S}(N)=\prod_{n=1}^{N} U_{S}(n)=U_{S}(N) U_{S}(N-1) \ldots U_{S}(1),
$$

can be represented in any of the forms above. In particular, for any $n$ the normal ordered form,

$$
U_{S}(n)=\exp \left[A_{+}(n) S_{+}\right] \exp \left[A_{0}(n) S_{0}\right] \exp \left[A_{-}(n) S_{-}\right],
$$

can be obtained recursively using the quantities $A_{ \pm, 0}(n-1)$ defined at the previous discretization step $n-1$ and the quantities $a_{ \pm, 0}(N)$ defined at the step $n$. The explicit formulas are stated in [46]. 


\section{Connection between group-theoretic and geometric approaches: Example of $\mathrm{SU}(2)$}

For the group $\mathrm{SU}(2)$ we define $\left[t^{a}, t^{b}\right]=i \epsilon^{a b c} t^{c}$. A basis for the algebra can be taken to be a set of Pauli matrices. We have for the arbitrary element of $g \in G, g^{-1} d g=$ $\sum_{a} \omega_{a} t_{a}$, where $\omega_{a}$ is the Maurer-Cartan form. These oneforms satisfy $d \omega_{a}+\frac{1}{2} f_{a b c} \omega_{b} \wedge \omega_{c}=0$, because of the identity $d\left(g^{-1} d g+g^{-1} d g \wedge g^{-1} d g=0\right.$. Parametrizing SU(2) as $\alpha=$ $\cos \left(u_{1} / 2\right) \exp \left[i\left(u_{2}+u_{3}\right) / 2\right]$ and $\beta=i \sin \left(u_{1} / 2\right) \exp \left[i\left(u_{2}-\right.\right.$ $\left.\left.u_{3}\right) / 2\right]$, the one-form becomes

$$
\frac{i}{2}\left(\begin{array}{cc}
d u_{3}+\cos u_{1} d u_{1} & e^{-i u_{3}}\left(d u_{1}+i d u_{2} \sin u_{1}\right) \\
e^{i u_{3}}\left(d u_{1}-i d u_{2} \sin u_{1}\right) & -d u_{3}-\cos u_{1} d u_{1}
\end{array}\right) .
$$

This means that

$$
\begin{aligned}
& \omega_{1}=\cos u_{3} d u_{1}+\sin u_{3} \sin u_{1} d u_{2}, \\
& \omega_{2}=\sin u_{3} d u_{1}-\cos u_{3} \sin u_{1} d u_{2}, \\
& \omega_{3}=\cos u_{1} d u_{2}+d u_{3} .
\end{aligned}
$$

Using these equations, one can readily check the MaurerCartan equation, $d \omega_{a}+\frac{1}{2} \epsilon_{a b c} \omega_{b} \wedge \omega_{c}$.

Geometrically speaking, $\mathrm{SU}(2)$ is equivalent to $S^{3}$. The metric induced on it by the embedding into $R^{4}=C^{2}$ can be found easily,

$$
d s^{2}=d u_{1}^{2}+d u_{2}^{2}+d u_{3}^{2}+2 \cos u_{1} d u_{2} d u_{3} .
$$

The determinant of the metric $\operatorname{det}\left(g_{\mu \nu}\right)=\sin ^{2} u_{1}$ and therefore the volume of the $S^{3}$ is normalized as $\int_{\mathrm{SU}(2)} \operatorname{det}^{1 / 2}\left(g_{\mu \nu}\right) d u_{1} d u_{2} d u_{3}=16 \pi^{2}$, the inverse of the metric yields

$$
g^{\mu \nu}=\left(\begin{array}{ccc}
1 & 0 & 0 \\
0 & \csc ^{2} u_{1} & -\cot u_{1} \csc u_{1} \\
0 & -\cot u_{1} \csc u_{1} & \csc ^{2} u_{1}
\end{array}\right),
$$

and nonzero Christoffel symbols read

$$
\begin{aligned}
& \Gamma_{23}^{1}=\frac{1}{2} \sin u_{1}, \quad \Gamma_{13}^{2}=\Gamma^{3} 12=\frac{-1}{2 \sin u_{1}}, \\
& \Gamma_{13}^{3}=\Gamma^{2} 12=\frac{1}{2} \cot u_{1} .
\end{aligned}
$$

The dreibein on $S^{3}$ is equal to $\omega$ within our normalization (otherwise there may arise a proportionality factor),

$$
e_{\mu}^{a}=\left(\omega_{a}\right)_{\mu}, \quad e^{a}=e_{\mu}^{a} d x^{\mu}=\omega_{a} .
$$

One can check that

$$
e_{\mu}^{a} e_{\nu}^{b} \eta_{a b}=g_{\mu \nu}
$$

The inverse dreibein is

$$
E_{a}^{\mu}=\eta_{a b} g^{\mu \nu} e_{\mu}^{b}
$$

and is used to define left-invariant vector fields

$$
l_{a}=E_{a}^{\mu} \frac{\partial}{\partial x^{\mu}}
$$

which in this case are

$$
\begin{aligned}
& l_{1}=\cos u_{3} \frac{\partial}{\partial u_{1}}+\frac{\sin u_{3}}{\sin u_{1}} \frac{\partial}{\partial u_{2}}-\sin u_{3} \cot u_{1} \frac{\partial}{\partial u_{3}}, \\
& l_{2}=\sin u_{3} \frac{\partial}{\partial u_{1}}-\frac{\cos u_{3}}{\sin u_{1}} \frac{\partial}{\partial u_{2}}+\cos u_{3} \cot u_{1} \frac{\partial}{\partial u_{3}}, \\
& l^{3}=\frac{\partial}{\partial u_{3}} .
\end{aligned}
$$

One can see that $e^{a}\left(l_{b}\right)=\delta_{b}^{a}$ and that these vector fields form the $\mathrm{su}(2)$ algebra themselves. The spin connection satisfies $d e^{a}+\omega_{b}^{a} \wedge e^{b}=0$ and is given explicitly by equations

$$
\begin{aligned}
\omega_{b \mu}^{a} & =-E_{b}^{\nu}\left(\partial_{\mu} e_{\nu}^{a}-\Gamma_{\mu \nu}^{\lambda} e_{\lambda}^{a}\right), \\
\partial_{\mu} e_{\nu}^{a} & =\Gamma_{\mu \nu}^{\lambda} e_{\lambda}^{a}-e_{\nu}^{b} \omega_{b \mu}^{a}, \quad \omega_{b}^{a}=\epsilon_{b c}^{a} e^{c} .
\end{aligned}
$$

The Laplace-Beltrami operator yields $\Delta_{\mathrm{LB}}=l_{a}^{2}$.

All the expressions above are representation dependent. In particular, in the representation defined by the Cartan decomposition we have

$$
\begin{aligned}
g_{G} & =e^{\psi S_{-}} e^{\phi S_{z}} e^{\chi S_{+}} \\
& =\left(\begin{array}{ll}
1 & 0 \\
\psi & 1
\end{array}\right)\left(\begin{array}{cc}
e^{\phi} & 0 \\
0 & e^{-\phi}
\end{array}\right)\left(\begin{array}{ll}
1 & \chi \\
0 & 1
\end{array}\right),
\end{aligned}
$$

and the Maurer-Cartan currents are given by

$$
\begin{aligned}
g^{-1} d g & =\left(\begin{array}{cc}
-e^{2 \phi} \chi d \psi+d \phi & -e^{2 \phi} \chi^{2} d \psi+2 \chi d \phi+d \chi \\
e^{2 \phi} d \psi & e^{2 \phi} \chi d \psi-d \phi
\end{array}\right), \\
d g g^{-1} & =\left(\begin{array}{cc}
-e^{2 \phi} \psi d \chi+d \phi & e^{2 \phi} d \chi \\
-e^{2 \phi} \psi^{2} d \chi+2 \psi d \phi+d \psi & e^{2 \phi} \psi d \chi-d \phi
\end{array}\right),
\end{aligned}
$$

while the left-invariant vector fields read

$$
\begin{aligned}
& l_{+}=\frac{\partial}{\partial \chi}, \quad l_{3}=-2 \chi \frac{\partial}{\partial \chi}+\frac{\partial}{\partial \phi}, \\
& l_{-}=e^{-2 \phi} \frac{\partial}{\partial \psi}+\chi \frac{\partial}{\partial \phi}-\chi^{2} \frac{\partial}{\partial \chi}
\end{aligned}
$$

and the right-invariant fields yield

$$
\begin{aligned}
& r_{+}=\frac{\partial}{\partial \psi}, \quad r_{3}=-2 \psi \frac{\partial}{\partial \psi}+\frac{\partial}{\partial \phi}, \\
& r_{-}=e^{-2 \phi} \frac{\partial}{\partial \chi}+\psi \frac{\partial}{\partial \phi}-\psi^{2} \frac{\partial}{\partial \psi} .
\end{aligned}
$$

The components of the vielbein can be read off these results straightforwardly. Validity of the Maurer-Cartan equation can also be checked. Also note that $\left(g^{-1} d g\right)^{a} r_{b}=\delta_{b}^{a}$, and $\left(d g g^{-1}\right)^{a} l_{b}=\delta_{b}^{a}$.

\section{Discretized version of the disentangling equations in the stochastic formulation}

If one wants to interpret the equations as stochastic differential equations with respect to the Wiener process, one should look into the exact discretization outlined in Eqs. (A22), and explicitly in Ref. [46]. Let us split $\phi_{ \pm, 3}$ into a white-noise and deterministic part, i.e., define $\phi_{ \pm, 3}=\sqrt{\varepsilon} \varphi_{ \pm, 3}+\varepsilon h_{ \pm, 3}=$ $d R_{ \pm, 3}+h_{ \pm, 3} d t$. For convenience, we denote the discretized versions of $\xi_{+}(t), \xi_{-}(t), \xi_{z}(t)$ as $A_{n}, C_{n}, B_{n}$, respectively. The 
discretized equations then take the following form:

$$
\begin{aligned}
A_{n+1}= & A_{n}+\sqrt{\varepsilon}\left(\varphi_{+, n}+A_{n} \varphi_{3, n}-A_{n}^{2} \varphi_{-, n}\right) \\
& +\varepsilon\left(h_{+, n}+A_{n} h_{3, n}-A_{n}^{2} h_{-, n}\right) \\
& +\frac{\varepsilon}{2}\left(\varphi_{+, n} \varphi_{3, n}+A_{n} \varphi_{3, n}^{2}-2 A_{n} \varphi_{+, n} \varphi_{-, n}\right. \\
& \left.-3 A_{n}^{2} \varphi_{3, n} \varphi_{-, n}+2 A_{n}^{3} \varphi_{-, n}^{2}\right)+o(\varepsilon), \\
C_{n+1}= & C_{n}+\sqrt{\varepsilon} e^{B_{n}} \varphi_{-, n}
\end{aligned}
$$
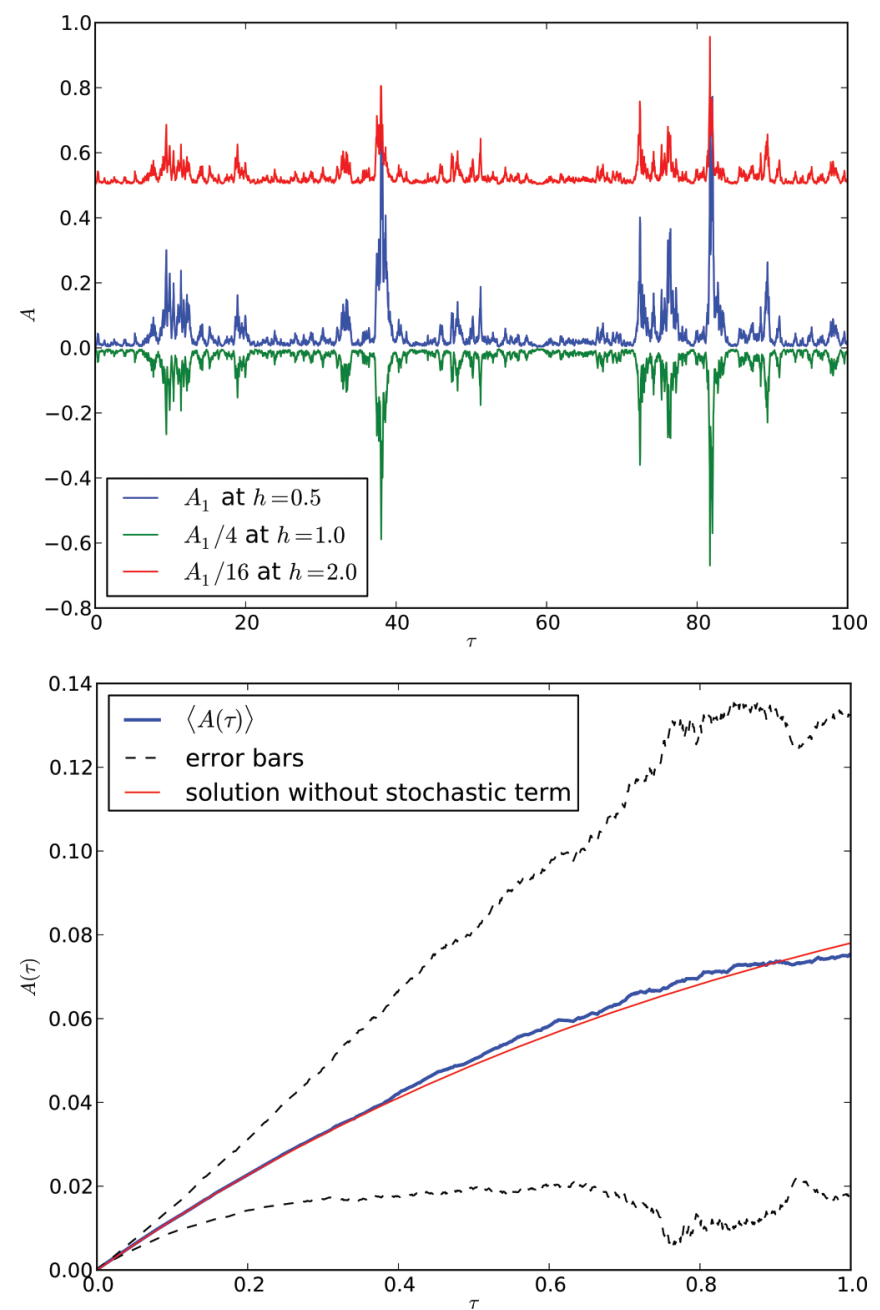

FIG. 3. (Color online) Simulation results for an Ising chain of 16 spins in a transversal magnetic field $h$ subject to periodic boundary conditions. The imaginary time $\tau$ (the magnetic field $h$ ) are measured in units of $J^{-1}(J)$, where $J$ is the nearest-neighbor hopping. The stochastic process $A$ itself is dimensionless. Upper panel: Typical realizations of the stochastic process $A_{1}(\tau)$, in imaginary time $\tau$ for various magnetic fields $h$. Values of $A_{1}$ for different $h$ are rescaled, mirrored, and shifted to improve their visibility. The magnitude of $A$ scales approximately as $h^{2}$. Trajectories are all taken for the same noise realization, so that one can compare the influence of a fluctuation in noise on the solution $A$ at different $h$. Lower panel: Initial part of the evolution of $A_{1}(\tau)$. The red line is a solution of the underlying SDEs neglecting the contributions of the noise term. The blue line is an average of solutions, averaged over 1000 realizations of the noise. Dashed lines represent error bounds given by $\sqrt{\operatorname{var} A(\tau)}$. We can clearly see the crossover from the "deterministic" region at small $\tau$ into a purely stochastic stationary regime at larger $\tau$.

$$
\begin{aligned}
& +\varepsilon e^{B_{n}}\left(h_{-, n}+\frac{1}{2} \varphi_{3, n} \varphi_{-, n}-A_{n} \varphi_{-, n}^{2}\right)+o(\varepsilon), \\
e^{B_{n+1}}= & e^{B_{n}}+\sqrt{\varepsilon} e^{B_{n}}\left(\varphi_{3, n}-2 A_{n} \varphi_{-, n}\right) \\
& +\varepsilon e^{B_{n}}\left(h_{3, n}-2 A_{n} h_{-, n}+\frac{1}{2} \varphi_{3, n}^{2}-\varphi_{+, n} \varphi_{-, n}\right. \\
& \left.-3 A_{n} \varphi_{3, n} \varphi_{-, n}+3 A_{n}^{2} \varphi_{-, n}^{2}\right)+o(\varepsilon) .
\end{aligned}
$$

These equations form the basis for the construction of the corresponding SDEs. The particular form of the equations clearly depends on the particular properties of the noises. For a general
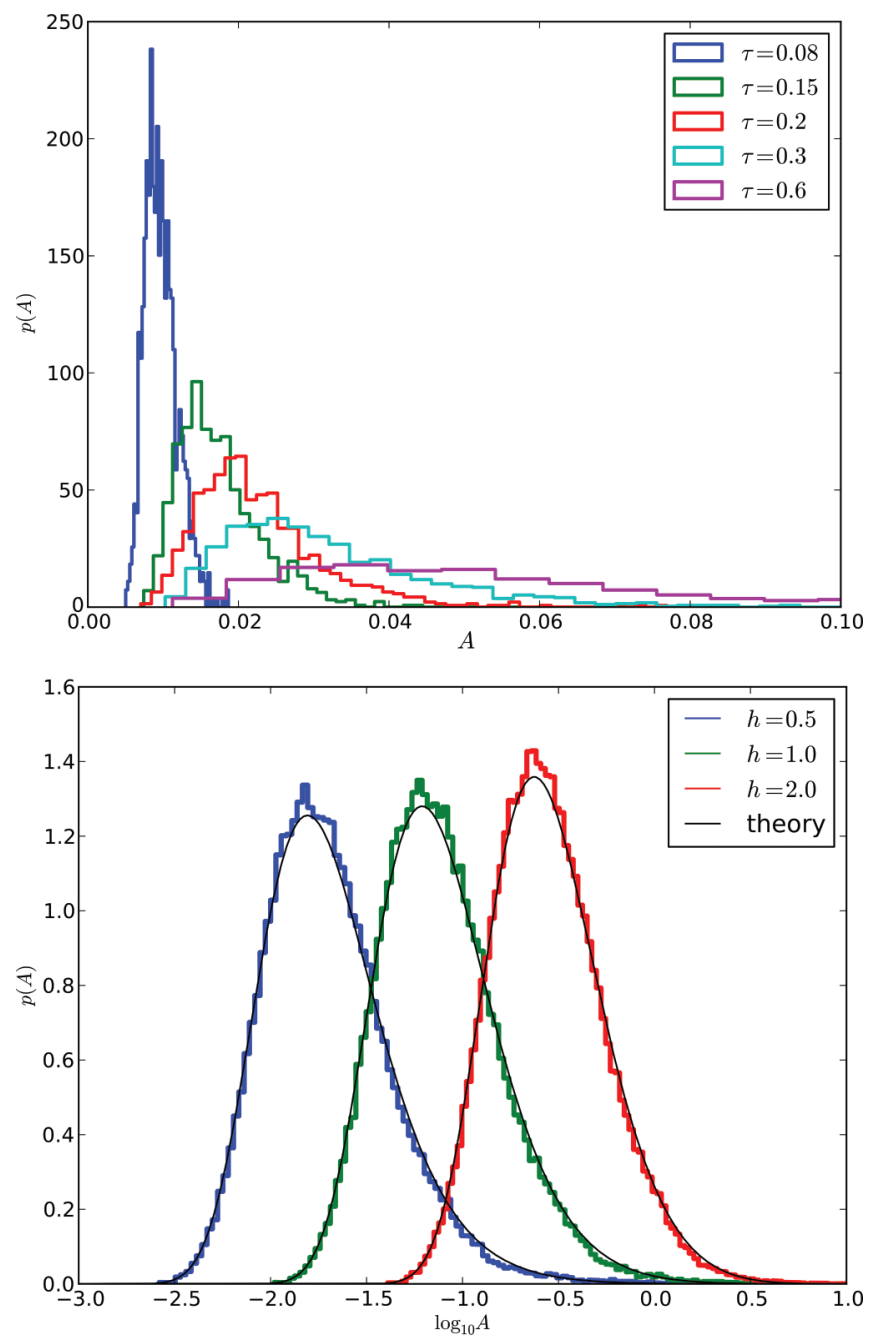

FIG. 4. (Color online) Simulation results for an Ising chain of 16 spins in a transversal magnetic field $h$ subject to periodic boundary conditions. The imaginary time $\tau$ (the magnetic field $h$ ) are measured in units of $J^{-1}(J)$, where $J$ is the nearest-neighbor hopping. The stochastic process $A$ itself is dimensionless. Upper panel: The time evolution of the probability distribution function $p\left(A_{1}\right)$ of $A_{1}(\tau)$. The histograms are plotted for 1000 realizations of the process with the magnetic field $h=0.5$. Lower panel: The empirical histograms of $A_{1}$ after the distribution becomes stationary (for large enough $\tau$ ) vs the asymptotic log-normal probability distribution, plotted for several magnetic fields $h$. Note that the horizontal axis is plotted in the log scale. The histograms shown in the left panel converge for large times $\tau$ to the ones in the lower panel. 
model with the quadratic coupling $\frac{1}{2} \Omega_{i j}\left(\gamma_{x} S_{i}^{x} S_{j}^{x}+\gamma_{y} S_{i}^{y} S_{j}^{y}+\right.$ $\left.\gamma_{z} S_{i}^{z} S_{j}^{z}\right)$ the noise action is $\frac{1}{2} \Omega_{i j}^{-1}\left(\gamma_{x}^{-1} \varphi_{i}^{x} \varphi_{j}^{x}+\gamma_{y}^{-1} \varphi_{i}^{y} \varphi_{j}^{y}+\right.$ $\left.\gamma_{z}^{-1} \varphi_{i}^{z} \varphi_{j}^{z}\right)$. The noises are thus $\varphi_{ \pm}=\frac{1}{2}\left(\varphi_{x} \mp i \varphi_{y}\right), \varphi_{3}=\varphi_{z}$. If the noises $d R_{ \pm, 3}$ are independent, we can rewrite the relations in the form of Ito SDE,

$$
\begin{aligned}
d A= & \left(h_{+}+A h_{3}-h_{-} A^{2}\right. \\
& \left.-\frac{K}{4} A\left[\gamma_{x}\left(1-A^{2}\right)+\gamma_{y}\left(1+A^{2}\right)-2 \gamma_{z}\right]\right) d t \\
& +\frac{1}{2}\left(1-A^{2}\right) d R_{x}-\frac{i}{2}\left(1+A^{2}\right) d R_{y}+A d R_{3}, \\
d C= & e^{B}\left(h_{-}-\frac{K}{4} A\left(\gamma_{x}-\gamma_{y}\right)\right) d t \\
& +\frac{1}{2} e^{B}\left(d R_{x}+i d R_{y}\right) \\
d e^{B}= & e^{B}\left(h_{3}-2 A h_{-}\right. \\
& \left.+\frac{K}{4}\left[2 \gamma_{z}+\gamma_{x}\left(3 A^{2}-1\right)-\gamma_{y}\left(3 A^{2}+1\right)\right]\right) d t \\
& +e^{B}\left[d R_{3}-A\left(d R_{x}+i d R_{y}\right)\right] .
\end{aligned}
$$

[1] H. Kleinert, Path Integrals in Quantum Mechanics, Statistics, Polymer Physics, and Financial Markets (World Scientific, Singapore, 2009).

[2] J. Zinn-Justin, Quantum Field Theory and Critical Phenomena (Oxford University Press, New York, 2002).

[3] J. W. Negele and H. Orland, Quantum Many Particle Systems (Westview Press, Boulder, CO, 1998).

[4] E. Fradkin, Field Theories of Condensed Matter Physics (Cambridge University Press, Cambridge, UK, 2013).

[5] V. I. Yudson and P. Reineker, Phys. Rev. A 78, 052713 (2008); V. I. Rupasov and V. I. Yudson, Zh. Eksp. Teor. Fiz. 87, 1617 (1984) [Sov. Phys. JETP 60, 927 (1984)]; Zh. Eksp. Teor. Fiz. 86, 819 (1984) [Sov. Phys. JETP 59, 478 (1984)]; V. I. Yudson, Zh. Eksp. Teor. Fiz. 88, 1757 (1985) [Sov. Phys. JETP 61, 1043 (1985)].

[6] E. Witten, Commun. Math. Phys. 117, 353 (1988); 118, 411 (1988).

[7] E. Frenkel, A. Losev, and N. Nekrasov, arXiv:hep-th/0610149; arXiv:hep-th/0702137; arXiv:0803.3302; A. Losev and S. Slizovskiy, J. Geom. Phys. 61, 1868 (2011).

[8] J. Hubbard, Phys. Lett. 3, 77 (1959).

[9] A. Perelomov, Generalized Coherent States and their Applications (Springer, New York, 1986).

[10] I. S. Burmistrov, Y. Gefen, and M. N. Kiselev, Pis'ma Zh. Eksp. Teor. Fiz. 92, 202 (2010).

[11] I. S. Burmistrov, Y. Gefen, and M. N. Kiselev, Phys. Rev. B 85, 155311 (2012).

[12] I. V. Kolokolov, Phys. Lett. A 114, 99 (1986).

[13] I. V. Kolokolov, Ann. Phys. (NY) 202, 165 (1990).

[14] M. Chertkov and I. V. Kolokolov, Phys. Rev. B 51, 3974 (1995).
Here we assume that the noises $d R$ can be written in terms of unit Wiener processes $d W$ via $d R_{x, y, z}=$ $\sqrt{\gamma_{x, y, z}} \sum_{i} T_{i l} d W_{l ; x, y, z}$. The constant $K$ in Eqs. (A38) is expressed via $T_{i j}$ as $K:=\sum_{l} T_{i l}^{2}$. The matrix $T_{i j}$ can be straightforwardly related to the inverse of the matrix $O_{i j}$ defined above Eq. (17). These equations can be cast into a much simpler form by utilizing the Stratonovich interpretation:

$$
\begin{aligned}
d A= & \left(h_{+}+A h_{3}-h_{-} A^{2}\right) d t+\frac{1}{2}\left(1-A^{2}\right) d R_{x} \\
& -\frac{i}{2}\left(1+A^{2}\right) d R_{y}+A d R_{3}, \\
d C= & e^{B}\left(h_{-}\right) d t+\frac{1}{2} e^{B}\left(d R_{x}+i d R_{y}\right), \\
d e^{B}= & e^{B}\left(h_{3}-2 A h_{-}\right) d t+e^{B}\left[d R_{3}-A\left(d R_{x}+i d R_{y}\right)\right] .
\end{aligned}
$$

Further insight into the SDE interpretation of the equations may be obtained from Figs. 3 and 4, where we present numerical results for a $1 \mathrm{D}$ Ising chain of $N=16$ spins in the transversal magnetic field $h$, simulated in the imaginary time $\tau$. These figures display typical trajectories of the local function $A_{1}$ (thanks to the translational invariance, we may choose any site), as well as its probability distribution. Let us note that the stochastic process $A$ becomes stationary at large imaginary times, obeying the log-normal distribution.
[15] M. Chertkov and I. V. Kolokolov, Sov. Phys. JETP 79, 824 (1994).

[16] I. V. Kolokolov, JETP 76, 1099 (1993).

[17] I. V. Kolokolov, Int. J. Mod. Phys. B 10, 2189 (1996).

[18] P. M. Hogan and J. T. Chalker, J. Phys. A: Math. Gen. 37, 11751 (2004).

[19] V. Galitski, Phys. Rev. A 84, 012118 (2011).

[20] M. Ringel and V. Gritsev, Europhys. Lett. 99, 20012 (2012).

[21] J. Wei and E. Norman, J. Math. Phys. 4, 575 (1963).

[22] V. G. Kac, Infinite-dimensional Lie Algebras (Birkhäuser, Boston, 1983).

[23] A. A. Kirillov, Elements of the Theory of Representations (Springer, New York, 1976).

[24] To shorten notations the explicit time arguments are sometimes suppressed for variables under the integration sign.

[25] C. S. Gardner, J. M. Greene, M. D. Kruskal, and R. M. Miura, Phys. Rev. Lett. 19, 1095 (1967).

[26] F. Langouche, D. Roekaerts, and E. Tirapegui, Functional Integration and Semiclassical Expansions (Reidel, Dordrecht, 1982); H. Calisto and E. Tirapegui, Phys. Rev. E 65, 038101 (2002)

[27] P. Arnold, Phys. Rev. E 61, 6091 (2000); 61, 6099 (2000).

[28] C. Becchi, A. Rouet, and R. Stora, Ann. Phys. (NY) 98, 287 (1976); I. V. Tyutin, Lebedev Physics Institute Report No. 39, arXiv:0812.0580.

[29] G. Parisi and N. Sourlas, Nucl. Phys. B 206, 321 (1982).

[30] M. V. Feigelman and A. M. Tsvelik, JETP 83, 1430 (1982).

[31] D. Hochberg, C. Molina-Paris, J. Perez-Mercader, and M. Visser, Phys. Rev. E 60, 6343 (1999); K. Mallick, M. Moshe, and H. Orland, J. Phys. A 44, 095002 (2011). 
[32] D. Friedan and A. Konechny, Adv. Theor. Math. Phys. 13, 1847 (2009).

[33] G. Parisi and N. Sourlas, Phys. Rev. Lett. 43, 744 (1979).

[34] I. V. Ovchinnikov, Chaos 22, 033134 (2012); 23, 013108 (2013).

[35] J.-T. Shen and S. Fan, Phys. Rev. A 76, 062709 (2007).

[36] T. Shi and C. P. Sun, Phys. Rev. B 79, 205111 (2009).

[37] T. Shi, S. Fan, and C. P. Sun, Phys. Rev. A 84, 063803 (2011).

[38] H. Lehmann, K. Symanzik, and W. Zimmermann, Nuovo Cimento 1, 205 (1955).

[39] In terms of the standard stochastic calculus the white noise $\Phi$ is related to the Wiener process $W$ as $W(t)-W(s)=\int_{s}^{t} d \tau \Phi(\tau)$ [40].

[40] C. W. Gardiner, Handbook of Stochastic Methods (SpringerVerlag, Berlin, 2004).
[41] Equations (7) are written in the Stratonovich form. We had to convert the equations into the Ito form, which is easier to work with.

[42] The Ito lemma establishes the rule for the change of variables in a SDE. If a collection of stochastic functions $x_{i}$ satisfy the SDEs $d x_{i}=A_{i} d t+B_{i} d W$, then according to the Ito lemma a function $f\left(x_{i}\right)$ satisfies the $\operatorname{SDE} d f=\left(\sum_{i} A_{i} \frac{\partial f}{\partial x_{i}}+\right.$ $\left.\sum_{i, j} \frac{1}{2} B_{i} B_{j} \frac{\partial^{2} f}{\partial x_{i} \partial x_{i}}\right) d t+\sum_{i} B_{i} \frac{\partial f}{\partial x_{i}} d W$.

[43] S. De Liberato, D. Gerace, I. Carusotto, and C. Ciuti, Phys. Rev. A 80, 053810 (2009).

[44] D. A. Fuhrmann et al., Nat. Photon. 5, 605 (2011).

[45] H. Touchette, Phys. Rep. 478, 1 (2009).

[46] M. Ban, J. Opt. Soc. Am. B 10, 1347 (1993). 